\title{
TOWARDS REMOTE MONITORING AND REMOTELY SUPERVISED TRAINING
}

Hermie J. Hermens

Roessingh Research and Development, Enschede, The Netherlands

Faculty of Electrical Engineering, Mathematics and Computer Science, University of Twente, Enschede, The Netherlands

The growing number of elderly and people with chronic disorders in our western society puts such pressure on our healthcare system that innovative approaches are demanded to make our health care more effective and more efficient. One way of innovation of healthcare can be obtained by introducing new services which enable less pressure on the intramural health care and support a more independent living and self efficacy of patients. Two of such services are Remote monitoring and remotely supervised training (RMT).

Remote monitoring enables freedom to the patient with the assurance that assistance is possible whenever required. Remotely supervised treatment enables efficient and effective user-centred training anywhere and anytime with an intensity not feasible in an intramural setting. It is our vision that remote monitoring and remotely supervised treatment applications will become very important for patients (safety, more in control, convenience), health care insurances (efficiency, cost reduction) and healthcare service providers (more effective, innovative).

A schematic drawing of the main components and the information exchange pathways are shown in the figure below. RMT systems are in general quite complex, requiring dependable end-to-end systems integrating ambulant sensing of relevant biosignals and context information, an M-Health platform for a secure data transport and storage and a backend system with appropriate decisions support with respect to both technical and clinical aspects. Another key element is the feedback of the information (dotted lines), which is essential for the subject and the care providers to learn from the data and to make the appropriate decisions.

Feedback

Sensing

Hospital

Informal coach

Decision support

\&

Central Database

UGATPDMRSSLS/I

Care \&

Coaching

During the past years considerable knowledge and experience is being gained with the research and development of such smart systems. In parallel, experience is gained with respect to the implementation of RMT systems in a clinical environment and how to assess its potential benefits. Examples concern: Activity monitoring in low back pain: the daily activity patterns of patients with chronic low back pain have been assessed, indicating a similar amount of total daily activity compared to healthy subjects but with a different distribution over the day. The results clearly show in general a higher activity level in the morning and lower activity levels in the afternoon and evening. This provides a starting point for treatment: restore the balance in acivity pattern by providing adequate personalised feedback

Monitoring of spasticity: continuous monitoring of muscle activity patterns by means of surface EMG measurement, in subjects with a spinal cord lesion provides insight in the magnitude and distribution of spasticity over the day. It gives direct objective and quantitative information during which activities and during which part of the day spasticity is most hindering the activities of daily living and as such it provides valuable information to optimise the drug delivery to decrease spasticity.

Myofeedback in subjects with neck-shoulder and low back pain. An ambulatory myofeedback treatment was developed which involves measurement of the muscle activation of neck/shoulder muscles and providing feedback by means of vibration when relaxation in these muscles is insufficient. Clinical trials showed that this treatment works well but to make the treatment more efficient, a service is set-up to enable remote monitoring of the muscle activation patterns and remote consultation. As a first step in the development of a myofeedback treatment for low back pain, low back muscle activation patterns are monitored. Based on the patterns a specific ambulatory feedback program is being developed to normalise the muscle activation patterns

55

Post rehabilitation home training (EC project HelloDoc). A modular system was developed to enable a wide range of upper extremity exercises at home, which can be monitored at a distance. In an international study it was shown that the effects were very similar to the traditional intramural treatment. There was also a clear indication that subjects who train more, do improve more. This underlines the potential that with this kind of training, the patient is in the driver seat and can significantly influence the results of his training.

Regarding the present status of remotely supervised treatment one could state that until now the focus has been on the technical realisation of the sensing and transportation part of it. The development of a backend system with an appropriate decision support system is still in its infancy as well as models how to organise these services and how to make them profitable. Probably most important is to gain more experience in this area in a realistic healthcare setting. Our experience gained in the past years in a rehabilitation setting, has learned us that this is a way forward with great potential to increase both the quality of treatment as well as its efficiency. It requires however a step-by-step approach with a strong and continuous involvement of a multidisciplinary team to guarantee a successful implementation. 